\title{
Determinação da densidade de líquidos imiscíveis pelo princípio de Stevin
}

\author{
Density Determination of immiscible liquids by the Stevin's principle
}

\author{
Andreia Vaz Gomes ${ }^{1}$, Elessandra Martins de Souza Amaral ${ }^{1}$, Rogério Junqueira Prado*1@ \\ ${ }^{1}$ Universidade Federal de Mato Grosso, Instituto de Física, Cuiabá, MT, Brasil
}

Recebido em 09 de Novembro, 2018. Revisado em 21 de Novembro, 2018. Aceito em 22 de Novembro, 2018.

\begin{abstract}
Este artigo apresenta uma sequência didática acompanhada por um aparato experimental de baixo custo e fácil montagem e manuseio, a serem aplicados ao estudo da hidrostática e, particularmente, de uma composição de líquidos imiscíveis, como a água e o óleo. O Princípio de Stevin é utilizado para analisar a densidade de líquidos, tendo como referência a densidade da água $\left(\rho=1000 \mathrm{~kg} / \mathrm{m}^{3}\right)$. O professor nesta proposta tem papel fundamental, pois ele é o mediador de todo processo, para assim construir a relação entre os conceitos já existentes na estrutura cognitiva dos alunos com os novos temas. Os conteúdos físicos foram fundamentados a partir de aula expositiva, discorrendo sobre seus conceitos, história e aplicações no cotidiano, de forma a organizar os conhecimentos prévios dos alunos. Por fim, foi feita a reconciliação integrativa do conceito durante a experimentação, de maneira a melhor proporcionar aprendizagem significativa. Com os resultados, além de observar que, num recipiente qualquer, líquidos de menor densidade se posicionam sobre líquidos de maior densidade, os alunos também determinam a densidade de líquidos imiscíveis, obtendo valores similares aos encontrados na literatura. Por exemplo, determinou-se a densidade do óleo de soja como sendo de $0,87 \mathrm{~g} / \mathrm{cm}^{3}$, sendo que o valor aceito é de $0,89 \mathrm{~g} / \mathrm{cm}^{3}$, uma diferença de cerca de $2 \%$.
\end{abstract}

Palavras-chave: Aprendizagem significativa, Líquidos imiscíveis, Experimentação, Princípio de Stevin.

\begin{abstract}
This work presents a didactic sequence followed by a low cost, easy to set up and easy to operate, experimental apparatus applied to the study of hydrostatics and, particularly, of a composition of immiscible liquids like water and oil. The Stevin Principle is used to analyze density of such liquids, having density of water $\left(\rho=1000 \mathrm{~kg} / \mathrm{m}^{3}\right)$ as reference. The teacher has a fundamental role in this proposal, since he is the mediator of the whole process, allowing the construction of connections between the existing concepts in student's cognitive structure with the new themes. The physical concepts were built through an expositive class, discussing on the physical concepts, history and everyday applications, as a form to better organize the previous knowledge of the students. Finally, an integrative reconciliation was made during the experimentation, intended to better provide meaningful learning. With the results, besides the observation that, in any given recipient, lower density liquids positionate itself above higher density ones, the students also determine the densities of such liquids, obtaining values similar to those found in literature. For example, the density of soy oil was determined as $0,87 \mathrm{~g} / \mathrm{cm}^{3}$ using the proposed experiment, against an accepted value of $0,891 \mathrm{~g} / \mathrm{cm}^{3}$, a difference of about $2 \%$.
\end{abstract}

Keywords: Meaningful Learning, Hydrostatic, Experimentation, Stevin's Principle.

\section{Introdução}

Muitas vezes, as aulas de Física são consideradas pacatas e sem graça, sendo ministradas de forma tradicional, com o uso de quadro e giz. Não que essa metodologia de aula esteja essencialmente errada mas, assim como quando se lê uma história ou um conto instigante, contagiante, o professor, como um mediador, deve motivar os alunos e conduzi-los por caminhos que despertem sua imaginação, instigando-os no agouroço da curiosidade, transformandoos em questionadores e investigadores de fenômenos, e inspirando-os a se arriscar por lugares sombrios e úmidos para encontrarem, não somente através das páginas gastas dos livros, a representação, contextualização, explica-

*Endereço de correspondência: rjprado@ufmt.br ção e compreensão de fenômenos naturais que moldaram a Física como uma ciência teórico-experimental fundamental, muito presente em nossa vida diária, e uma das mais belas construções humanas.

A Física, por ser uma ciência fundamental que abrange conteúdos muito amplos e variados é, por conseguinte, dividida em vários ramos para seu melhor estudo. Um deles é a hidrostática, que estuda os líquidos em equilíbrio estático, ou seja, que não estejam em movimento, relacionando a pressão e o equilíbrio dos líquidos que se submetem à ação gravitacional. Alguns exemplos comuns de sistemas hidrostáticos são: sistema de tubulações e caixa d'água em residências, nivelamento na construção civil e na agricultura dentre outros. 
Muitos físicos contribuíram para o estudo da hidrostática, entre eles podemos citar: Arquimedes (287-212 a.C.), que foi responsável por várias invenções, é famoso pela sua descrição do empuxo através do "Princípio de Arquimedes"; Simon Stevin (1548-1620), que demonstrou que a pressão que um líquido exerce sobre uma superfície depende apenas da altura da coluna do líquido e da área da superfície, não importando o tamanho ou a forma do recipiente. Ainda temos Blaise Pascal (1623-1662), que teve um papel importante nesse ramo da Física, explicando como se dá a transmissão de pressão num líquido através do "Princípio de Pascal".

Segundo a SEDUC-MT [1], o professor tem a função de formar cidadãos pensantes/críticos e ativos, atores de sua própria história, para que os mesmos possam futuramente contribuir de alguma forma para o crescimento e aprimoramento científico. E para que isso se concretize o professor deve fazer uso de abordagens e estratégias de ensino adequadas.

\section{“... o que a Física deve buscar no ensino médio é assegurar que a competência investigativa resgate o espírito questionador, o desejo de conhecer o mundo em que se habita". [1]}

Uma das possibilidades para que isso ocorra é a elaboração de uma proposta de aula experimental, uma vez que, segundo Araújo \& Abib [2] a experimentação é uma proposta de aprendizagem na qual há motivação por parte dos alunos, pois mesmo não gostando muitas vezes da disciplina, a grande maioria dos alunos gosta de ir para o laboratório realizar experimentos, proporcionando assim uma ferramenta facilitadora de aprendizagem. Ainda, Villani \& Nascimento [3] relatam que o laboratório proporciona aos alunos a utilização de argumentação e metodologias específicas do ensino de ciências, fazendo com que os mesmos passem a observar o cunho científico proposto em cada experimento.

O fato do aluno poder manusear e auxiliar na construção do aparato, não somente favorece mas também enriquece a aprendizagem, dando maior significado aos conteúdos abordados, permitindo a obtenção de um contexto, ambiente e/ou situações mais propícios(as) à aprendizagem, fazendo com que os alunos se interessem sobre o que está sendo proposto e, neste caso, tornando a abordagem experimental fundamental para a construção do conhecimento, ampliando as possíveis indagações, reflexões e conclusões dos alunos referente ao tema abordado $[3]$.

Assim, pensando em desviar do tradicionalismo, fazer algo mais do que apenas um roteiro experimental, e adotando os conceitos abordados como organizadores prévios, a proposta deste artigo é trabalhar a hidrostática através de uma sequência didática, com aula expositiva e também montagem de um aparato experimental de baixo custo (um tubo em U), que possibilite determinar a densidade de líquidos imiscíveis através do Princípio de Stevin. Será também possível observar que líquidos imis- cíveis colocados no mesmo recipiente não se misturam, se dispondo de forma que o líquido de maior densidade ocupe a parte inferior e o líquido de menor densidade ocupe a parte superior.

O livro de Young \& Freedman [4] foi utilizado como principal ferramenta de apoio para este trabalho, mas também foram utilizados outros livros didáticos $[5,6]$, artigos e teses, como por exemplo o artigo de Jesus \& Palma [7] que discutem as incertezas envolvidas no cálculo da densidade do óleo de cozinha por dois métodos, pelo cálculo direto da razão entre massa e volume e com o uso do tubo em U, propondo melhorias neste segundo método e buscando fomentar a busca por precisão e a análise, por parte dos futuros professores, de configurações variadas para a execução de experimentos em sala de aula. Vale ressaltar que um procedimento experimental bem executado deve levar à obtenção de dados compatíveis com a realidade, e que isto é fundamental para que o mesmo seja mais bem aceito e, consequentemente, mais relevante perante os alunos. Ao contrário, procedimentos mal executados, mal explicados, e/ou que obtenham informações duvidosas ou incompatíveis, não contribuirão de maneira significativa para o aprendizado do aluno, podendo inclusive prejudicá-lo.

Podemos ainda citar o roteiro experimental de Garcia et al. [8], que serviu como trabalho de base, mas ao qual buscou-se diferir através do desenvolvimento de uma sequência didática mais ampla e completa, embasada em teoria de aprendizagem e aplicação dos conceitos físicos a partir do aparato experimental, e que ainda proporcione discussões sobre os temas abordados através de atividades de estudo dirigido.

\section{Fundamentação teórica}

Segundo a teoria cognitiva de David Ausubel apud Moreira (2006) [9] o aluno deve estar envolvido no processo de aprendizagem, pois partimos do conhecimento prévio do mesmo promovendo relações entre os conceitos que ele já possui com novos, que no nosso caso são científicos, dando suporte para que esses novos conceitos se ancorem na estrutura cognitiva já existente, proporcionando assim aprendizagem.

Moreira [10] também cita que aprendizagem significativa é:

"...aquela em que ideias expressas simbolicamente interagem de maneira substantiva $e$ não-arbitrária com aquilo que o aprendiz já sabe. Substantiva quer dizer não-literal, não ao pé-da-letra, e não-arbitrária significa que a interação não é com qualquer ideia prévia, mas sim com algum conhecimento especificamente relevante já existente na estrutura cognitiva do sujeito que aprende".

Para que possamos relacionar os conceitos já existentes devemos ofertar atividades diversificadas, nas quais o 
aluno possa desenvolver os novos conceitos, ampliando seus conhecimentos. Desta forma, para que as diferentes atividades tenham sucesso, elas devem ser cuidadosamente planejadas, de maneira a disponibilizar as ferramentas e meios necessários para que proporcionem aprendizagem significativa.

Outra ferramenta utilizada para facilitar a aplicação de propostas educacionais são as sequências didáticas, que devem oferecer atividades variadas com o intuito de consolidar a aprendizagem e possibilitar a efetiva validação do conhecimento na estrutura cognitiva do aluno. As sequências didáticas devem basear-se nos conhecimentos prévios e proporcionar ampliação dos conhecimentos já existentes. Além disso [11], é fundamental o diálogo entre os alunos, professores e conhecimento, o que só será possível com a devida contextualização e utilização de objetos e fenômenos que façam parte do universo vivencial do aluno.

Nesse sentido, não somente a discussão de situações cotidianas é relevante, mas também a presença da experimentação no processo de aprendizagem da Física é de fundamental importância [11], uma vez que esta última possibilita o desenvolvimento das competências em Física, privilegiando-se o fazer, manusear, operar, agir, compreender, descrever em diferentes formas e níveis, favorecendo a construção do conhecimento pelo próprio aluno, desenvolvendo sua curiosidade e hábito de indagação, evitando a aquisição do conhecimento científico como uma verdade estabelecida e inquestionável. Para isso, todavia, deve-se evitar experimentos que se reduzam à execução de uma lista de procedimentos cujo sentido não fica claro para o aluno [11].

\subsection{Conceitos físicos}

A densidade de um corpo é uma propriedade importante do material que fornece sua quantidade média de massa por unidade de volume, ou seja é o grau de concentração de massa por unidade de volume. Logo,

$$
d=\frac{m}{V}
$$

Sendo $m$ a massa do corpo e V seu volume.

Embora muitas vezes densidade e massa específica sejam tratadas de forma análoga, há diferença entre essas grandezas que são importantes de serem ressaltadas pois, enquando a densidade é uma propriedade média do corpo, a massa específica é uma propriedade da substância, ou seja, para objetos ocos por exemplo, massa específica e densidade possuem valores muito diferentes. Massa específica é dada por:

$$
\rho=\frac{\Delta m}{\Delta V}
$$

Onde $\Delta m$ é a massa de um corpo ou substância homogêneo(a) contida em um volume $\Delta V$ que é totalme preenchido pela substância.
Outra grandeza importante para o estudo dos fluidos é a pressão, que em um fluido em repouso é dada pela razão entre a força exercida perpendicularmente sobre qualquer superfície que esteja em contato com o fluido, tal como a parede de um recipiente ou um corpo imerso no fluido, e a area dessa superfície. Logo:

$$
P=\frac{F_{\perp}}{A}
$$

Embora, macroscopicamente, o fluido como um todo esteja supostamente em repouso, microscopicamente as moléculas constituintes do fluido estão em movimento, e as forças exercidas pelo fluido sobre superfícies que estejam diretamente em contato com ele são oriundas de um processo contínuo de colisões de um gigantesco número de moléculas do fluido com essas mesmas superfícies.

A pressão nos permite entender muitos fenômenos físicos de nosso cotidiano, como cortar um pedaço de pão e cravar um prego em uma tábua, e também explica o motivo pelo qual precisamos fazer dois furos em uma lata para poder escoar o líquido que se encontra dentro dela

Quando se despreza a influência da gravidade sobre o fluído, a pressão no interior do fluído é a mesma em todos os pontos do seu volume. Porém, um fluido possui massa específica não nula e, consequentemente, estando ele na superfície da Terra, a influência da gravidade sobre ele não será desprezível. Daí a utilidade do Princípio de Stevin, que relaciona a pressão em um determinado ponto de um fluido com sua massa específica, a gravidade e a profundidade com relação a superfície

$$
P_{R}=P_{a t m}+\rho g \Delta h
$$

O princípio de Stevin diz literalmente que a pressão em um ponto situado a uma profundidade $\mathrm{h}$ no interior de um líquido em equilíbrio é dada pela pressão exercida pela coluna de líquido situada acima daquele ponto $(\rho g h)$ mais a pressão atmosférica exercida sobre a superfície $\left(P_{a t m}\right)$ do líquido.

As aplicações do Princípio de Stevin, são muitas, mas pode-se destacar a aplicação em vasos comunicantes, que permite explicar, por exemplo, o funcionamento de sistemas hidráulicos simples, como o residencial (tubulações e caixa d'água, estas últimas situadas no ponto mais alto das casas); ou até mesmo do vaso sanitário. Também é preciso compreender porque a pressão atmosférica em altitudes elevadas é menor que a pressão atmosférica ao nível do mar, e o porque do aumento da pressão, e de boa parte dos riscos envolvidos, em função da profundidade de um mergulho.

\subsection{Entendendo o princípio de Stevin}

A partir da figura 1, observando que o líquido do recipiente está submetido na sua superfície à pressão atmosférica, e utilizando o Princípio de Stevin, equação (4), podemos escrever a pressão hidrostática para cada um 


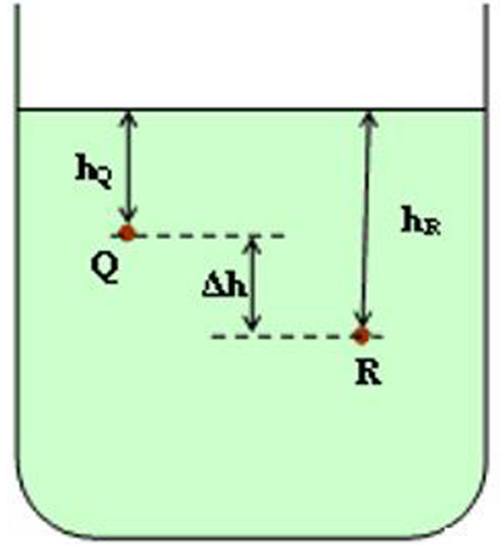

Figura 1: llustração dos pontos $Q$ e $R$ abaixo da superfície de um fluido contido num recipiente qualquer.

dos pontos Q e R como segue:

$$
p_{Q}=p_{a t m}+\rho g h_{Q}
$$

e

$$
p_{R}=p_{a t m}+\rho g h_{R},
$$

De maneira que a diferença de pressão entre esses dois pontos é:

$$
\begin{array}{r}
p_{R}-p_{Q}=\rho h_{R} g-\rho h_{Q} g \\
p_{R}-p_{Q}=\rho g\left(h_{R}-h_{Q}\right) \\
p_{R}-p_{Q}=\rho g(\Delta) h
\end{array}
$$

Isolando um dos pontos vamos ter:

$$
p_{R}=p_{Q}+\rho g \Delta h
$$

Assim pode-se chegar à conclusão de que se os pontos estiverem no mesmo nível $(\Delta h=0)$, em um líquido em equilíbrio, atua sobre eles a mesma pressão. De maneira que, aplicando esta situação para o objeto de estudo, por exemplo o tubo em U ilustrado na figura 2 , tem-se que $P_{C}=P_{D}$

Como $P_{A}=P_{B}=P_{a t m}$ temos também que $P_{C}=P_{D}$ (dois pontos no mesmo líquido e na mesma profundidade, $\log \mathrm{O}$

$$
\begin{gathered}
P_{C}=P_{A}+\rho_{\text {água }} g h_{\text {água }}=P_{a t m}+\rho_{\text {água }} g h_{\text {água }} \\
P_{D}=P_{B}+\rho_{\text {óleo }} g h_{\text {óleo }}=P_{a t m}+\rho_{\text {óleo }} g h_{\text {óleo }} \\
P_{c}=P_{D} \Leftrightarrow P_{a t m}+\rho_{\text {água }} g h_{\text {água }}=P_{a t m}+\rho_{\text {óleo }} g h_{\text {óleo }} \\
\rho_{\text {água }} h_{\text {água }}=\rho_{\text {óleo }} h_{\text {óleo }}
\end{gathered}
$$

e consequentemente

$$
\rho_{\text {óleo }}=\frac{\rho_{\text {água }} \cdot h_{\text {água }}}{h_{\text {óleo }}}
$$

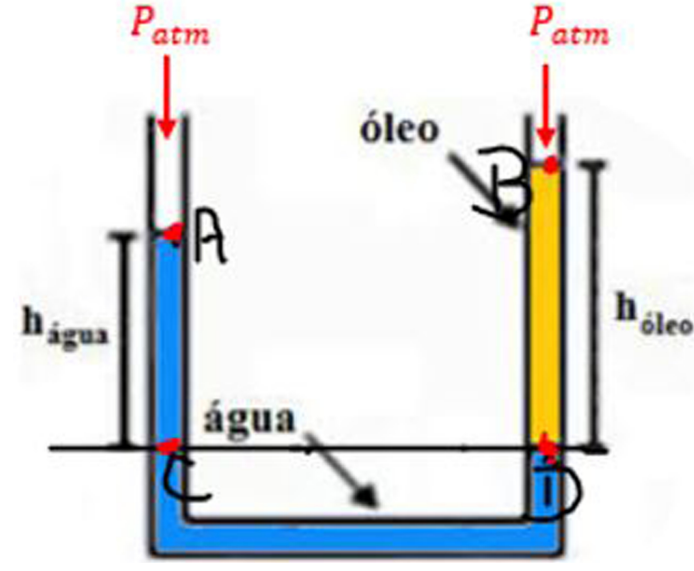

Figura 2: Ilustração de um tubo em U com dois fluidos imiscíveis.

\section{Materiais utilizados:}

1. Uma tábua de madeira que comporte o tubo em U, conforme figura 3 .

2. Uma folha milimétrica para registro das medidas.

3. Uma mangueira transparente de comprimento e espessuras proporcionais a madeira, conforme figura 3.

4. Duas abraçadeiras para fixação da mangueira na madeira.

5. Chaves de fenda.

6. Chaves Phillips.

7. Dois suportes de madeira perfuradas com o auxílio de uma furadeira.

8. Três bases de madeira.

9. 15 Parafusos.

10. 1 Régua.

11. 2 Conexões para mangueiras.

12. Papel contact.

13. Água.

14. Óleo.

\section{Metodologia}

Partindo do princípio no qual a aprendizagem significativa deve proporcionar ao aluno formas diversificadas para aprimorar seus conhecimentos prévios, propõe-se uma sequência didática com atividade experimental, que tem como objetivo trabalhar a hidrostática utilizando o Princípio de Stevin para determinar a densidade de líquidos imiscíveis. Para tanto, segue abaixo uma sugestão de aplicação da mesma em três aulas, que também é apresentada de maneira resumida na Tabela 1.

\subsection{Detalhamento das atividades}

\subsubsection{1 ${ }^{\mathrm{a}}$ aula : Aula expositiva.}

Propõe-se que os conceitos físicos sejam fundamentados a partir de aula expositiva, procurando abordar tais con- 


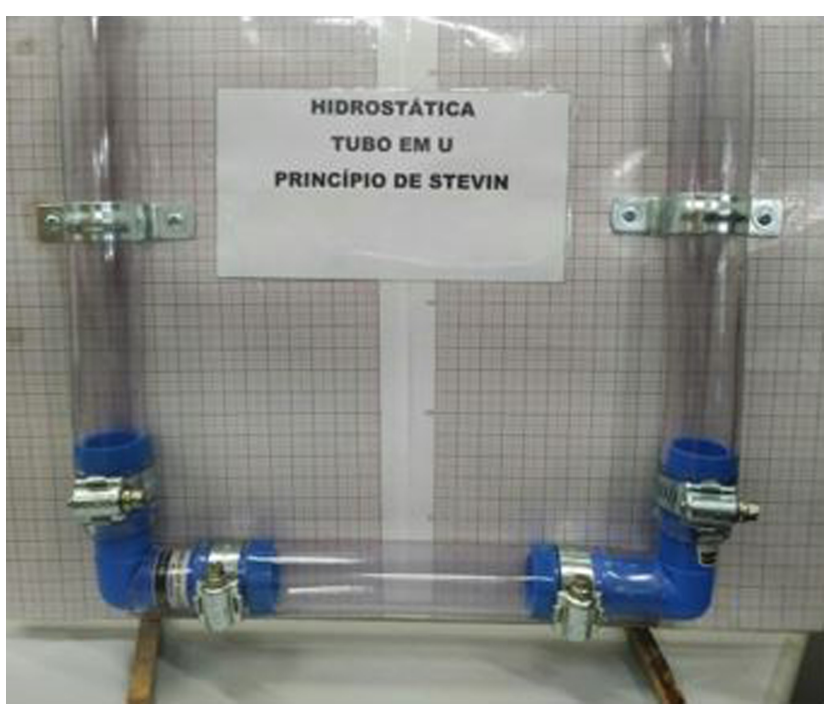

Figura 3: Tubo em U após a montagem.

ceitos de forma a servirem de organizadores prévios para o posterior experimento. Nesta etapa é fundamental que os mesmos sejam trabalhados de maneira a possibilitar ao aluno o desenvolvimento de uma relação entre teoria e prática vivenciada em sala de aula e no seu cotidiano. Ao final da aula, sugere-se que a turma seja dividida em grupos de três ou quatro alunos, e que seja divulgado o material necessário para a montagem do tubo em U. Esta divisão se faz necessária para uma maior integração e auxílio entre os membros do grupo, mas também em função do custo dos materiais, que muito provavelmente será dividido entre os alunos.

\subsection{2. $2^{\mathrm{a}}$ aula: Montagem do experimento.}

Para a montagem do experimento, cada grupo deverá cortar a mangueira em três partes, que serão ligadas com auxilio das conexões em joelho e abraçadeiras para formar o tubo em U. Note que seria possível elaborar um tubo em U sem as conexões, mas exigiria uma maior altura do aparato. Em seguida forra-se a madeira com a folha milimetrada e encapa-se a mesma com papel contact. Feito isto, basta fixar o tubo um U na madeira, com o uso das abraçadeiras, conforme ilustra a figura
3. O professor deve acompanhar cada passo para que os grupos consigam realizar a construção do tubo em U de forma adequada, rapidamente e sem desperdício de material.

\subsection{3. $3^{\text {a }}$ aula: Realizaçao da experimentação}

Como o experimento funciona como um reconciliador integrativo dos conceitos físicos abordados na aula expositiva, para intencionalmente proporcionar aprendizagem significativa, deve-se ter cuidado com a execução de todo e qualquer procedimentos no momento da aplicação. Particularmente, recomenda-se que o professor execute todos os procedimentos previamente à aplicação do mesmo em sala de aula, para que não restem dúvidas quanto aos mesmos. Para a experimentação, pede-se que os alunos despejem os líquidos no tubo, façam os registros das alturas ( $h_{\text {água }}$ e $h_{\text {óleo }}$ ) encontradas e então realizem o cálculo da densidade do óleo através do Princípio de Stevin. Nessa etapa é desejável que o professor justifique para os alunos o porquê da escolha de óleo e água, e explique características da estrutura das moléculas de cada substância utilizada, fundamentando e aprofundando os conceitos abordados [3]. Também é necessário que haja precisão na retirada dos dados, para que os resultados obtidos sejam compatíveis com os dados tabelados das densidades de cada tipo de óleo, considerando as margens de erro experimentais. Quanto maior a precisão dos valores encontrados, maior será a confiança dos alunos no conteúdo abordado e, consequentemente, maior o êxito do processo de experimentação.

Para exemplificar o cálculo a ser realizado, foi desenvolvido um exemplo didático (vide Anexo A) no qual os alunos desenvolverão as habilidades necessárias para a realização do mesmo.

Após a realização do experimento, sugere-se uma atividade dirigida, como a que se encontra no anexo B, para auxiliar na generalização dos conceitos e melhor fundamentar a proposta, dando mais significado ao que se quer ensinar.

Ainda sugerimos que o professor deixe como desafio aos grupos pesquisarem sobre outras aplicações do Princípio de Stevin, como o funcionamento dos vasos sanitários,

Tabela 1: Sequência didática.

\begin{tabular}{|c|c|c|c|c|c|c|}
\hline ETAPA & ATIVIDADE & DESCRIÇĀO & PRODUÇĀO & FINALIDADE & TEMPO & AULA \\
\hline I & Aula expositiva & $\begin{array}{l}\text { Apresentação dos concei- } \\
\text { tos físicos. }\end{array}$ & & $\begin{array}{l}\text { Aprendizagem representa- } \\
\text { cional/ organizador prévio }\end{array}$ & $55 \mathrm{~min}$. & 1 \\
\hline II & Aula prática & Construção do tubo em U. & & Assimilação & $55 \mathrm{~min}$. & $*_{2}$ \\
\hline III & Aula prática & $\begin{array}{l}\text { Realizar a experimenta- } \\
\text { ção. }\end{array}$ & $\begin{array}{l}\text { Desenvolver o cálculo da } \\
\text { densidade do óleo }\end{array}$ & Registro de dados & $25 \mathrm{~min}$. & 3 \\
\hline IV & Avaliação & $\begin{array}{l}\text { Atividade de estudo diri- } \\
\text { gido }\end{array}$ & Realização da atividade & Reconciliação Integrativa & $30 \mathrm{~min}$. & \\
\hline
\end{tabular}


a distribuição de água nas residências e o nivelamento de paredes na construção cívil. Nos anexos C, D e E, propõe-se situações problema para a discussão desses temas.

\section{Resultados e discussões}

No momento da realização da experimentação deve-se ter-se cuidado na retiradas dos dados, pois na experimentação poderão ser observadas informações importantes que devem ser debatidas posteriormente. Quanto maior o cuidado e a precisão na montagem e realização das medidas, mais próximo o resultado obtido estará do valor tabelado para a densidade do óleo, fazendo com que consigamos sucesso na obtenção de resultados, dando maior significado aos conceitos físicos estudados e, também, à metodologia científica.

Abaixo, na Tabela 2, seguem resultados obtidos pelos autores quando da experimentação proposta para o experimento, ilustrada na figura 4.

A partir dos dados obtidos utilizando a equação (14) e o valor da densidade da água, que é de $1,00 \mathrm{~kg} / \mathrm{l}$ ou 1,00 $\mathrm{g} / \mathrm{cm}^{3}$, é possível encontrar a densidade do óleo.

$$
\begin{gathered}
\rho_{\text {óleo }}=\frac{\rho_{\text {água }} \cdot h_{\text {água }}}{h_{\text {óleo }}} \\
\rho_{\text {óleo }}=\frac{(1,00) \cdot(55)}{(63)}
\end{gathered}
$$

Tabela 2: Dados obtidos para a altura da coluna dos diferentes líquidos (água e óleo) medida a partir do ponto de interface entre os líquidos no tubo em $\mathrm{U}$.

\begin{tabular}{lc}
\hline Altura & $(\mathrm{mm})$ \\
\hline$h_{\text {água }}$ & 55 \\
$h_{\text {óleo }}$ & 63 \\
\hline
\end{tabular}

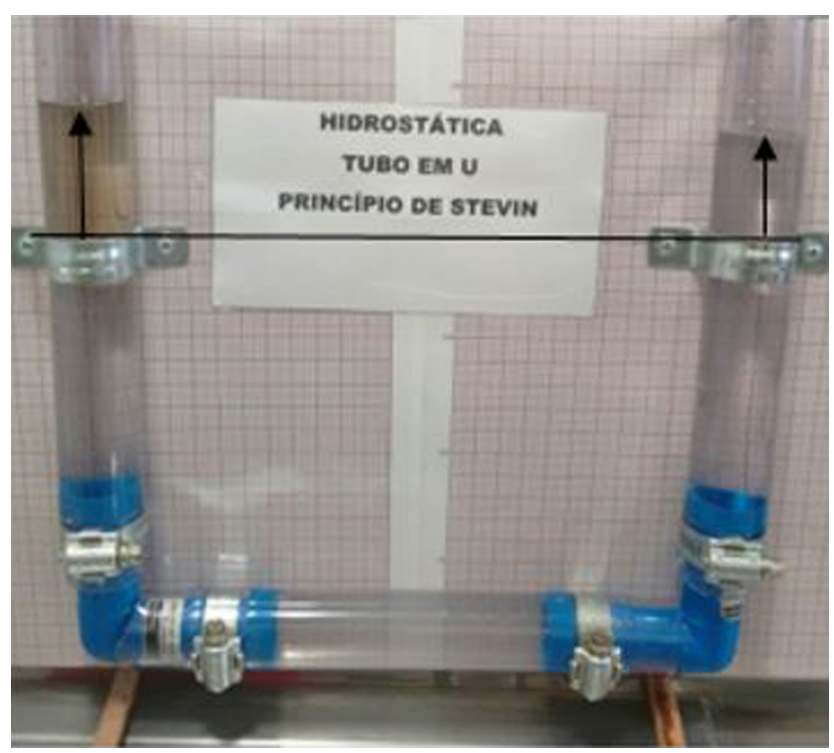

Figura 4: Ilustração do resultado do experimento.

$$
\begin{gathered}
\rho_{\text {óleo }}=\frac{55}{63} \\
\rho_{\text {óleo }}=0,87 \frac{\mathrm{g}}{\mathrm{cm}^{3}}
\end{gathered}
$$

O valor tabelado para a densidade do óleo de soja é 0,891 $\mathrm{g} / \mathrm{cm}^{3}$, de maneira que obtivemos um valor bem próximo ao esperado.

Alguns fatores podem influenciar a qualidade e/ou precisão dos dados obtidos, como a precisão com a qual as alturas das colunas de água e óleo são medidas no tubo em U, que deve estar exatamente na vertical, e a utilização de um óleo puro (de boa qualidade), pois muitas vezes o óleo possui impurezas ou misturas, fazendo com que o resultado obtido não seja compatível com o tabelado para o óleo puro. Esta seria, por exemplo, uma maneira de se verificar se o óleo de cozinha é de boa qualidade.

A verticalidade do papel milimetrado e dos tubos pode ser facilmente verificada com a utilização de um pequeno prumo ou mesmo colocando-se diferentes níveis de água no interior do tubo em U e ajustando a inclinação do sistema até que as medidas das posições dos diferentes níveis de água, obtidas com o auxílio do papel milimetrado, sejam idênticas em ambos os lados do tubo.

Uma vez que estes cuidados sejam levados em conta, o ideal é que se repita o experimento por, pelo menos, três vezes, para que se possa determinar um valor médio para a densidade do óleo e também se estimar o erro experimental da medida, possibilitando ao professor checar a precisão com a qual cada grupo realiza seu experimento e, consequentemente, identificar possíveis erros ou imprecisões nas medidas por eles realizadas.

Note que a precisão na determinação da densidade do óleo pode ser melhorada se utilizarmos tubos mais longos, como propõe Jesus \& Palma [7] após análise de propagação de erros, e consequentemente com a utilização de maiores quantidades de óleo e água. Deve-se tomar cuidado, todavia, com a utilização de tubos muito finos, pois nesse caso a capilaridade do óleo e da água podem influenciar na medida. Daí a escolha por tubos de maior diâmetro neste trabalho, o que possibilita que a medida da altura das colunas seja realizada no centro do tubo e com maior precisão.

Utilizando a propagação de erros, foi determinado que o erro experimental na medição realizada pelos autores é da ordem de $0,02 \mathrm{~g} / \mathrm{cm}^{3}$, considerando-se que o erro na medição das alturas das colunas não deve ser superior a $1 \mathrm{~mm}$ (se o aparato for corretamente montado). Erros experimentais maiores que este indicam que as medições não estão sendo bem realizadas pelo grupo, e que uma maior atenção por parte do professor é necessária. Note, todavia, que um erro na medida das alturas de $0,5 \mathrm{~mm}$ poderia ser obtido com uma medição muito bem executada, o que reduziria o erro experimental para a determinação da densidade do óleo para cerca de 0,01 $\mathrm{g} / \mathrm{cm}^{3}$. A mesma precisão poderia ser obtida dobrando-se 
o comprimento do tubo e, consequentemente, das colunas de água e óleo.

\section{Considerações finais}

O presente trabalho traz uma grande contribuição como ferramenta metodológica para aplicação de experimentação baseada na teoria de aprendizagem significativa de David Ausubel, através da utilização de um tubo em formato de U para a aplicação de conceitos de Hidrostática, possibilitando especificamente o cálculo da densidade de substâncias imiscíveis pela aplicação do Teorema de Stevin.

O tubo em U é comumente utilizado para o cálculo de pressão e também de densidades, porém nesta proposta evidencia-se a necessidade de unir aspectos quantitativos (experimentais) e qualitativos (teóricos) em sala de aula, sendo que ambos possuem papéis estratégicos importantes para sucesso da aplicação. Desta forma, o trabalho dá suporte ao professor para que este combine aula expositiva com experimentação, o que suscita a possibilidade de aplicação prática da teoria e também de discussão dos resultados encontrados em sala de aula, que podem ser confrontados com dados tabelados conhecidos, além de embasar outras aplicações do Princípio de Stevin.

Também se propõe, neste trabalho, um exemplo didático que possibilita desenvolver as habilidades e competências para o cálculo da densidade aqui proposto e, finalmente, atividades de estudo dirigido elaboradas para a aplicação, ampliação e generalização dos conceitos abordados em sala de aula, de forma a se potencializar a aprendizagem significativa dos conteúdos abordados.

Portanto, este trabalho é mais do que um mero roteiro experimental, mas uma proposta de ferramenta didática experimental diferenciada, baseada na teoria de aprendizagem significativa, e que leva em conta aspectos didáticos conceituais e práticos que visam não somente o aprendizado dos conceitos em si, mas também a divulgação e valorização da metodologia científica através de atividades práticas em sala de aula.

\section{Material suplementar}

O seguinte material suplementar está disponível online: ANEXO A - Proposta de exemplo didático a ser desenvolvido.

ANEXO B - Proposta de estudo dirigido para assimilação e generalização dos conceitos físicos.

ANEXO C - Princípio de Stevin e o vaso sanitário.

ANEXO D - Princípio de Stevin e a mangueira de nível. ANEXO E - Princípio de Stevin e a distribuição de água em nossas residências.

ANEXO F - Princípio de Stevin e o Mergulho.

\section{Referências}

[1] SEDUC-MT. Orientações Curriculares Área de Ciências da Natureza e Matemática (Defanti, Cuiabá, 2010).

[2] M.S.T. Araújo e M.L.V.S. Abib, Rev. Bras. Ens. Fís. 25, 19 (2003).

[3] C.E.P. Villani e S.S. Nascimento, Investigação em Ensino de Ciências 8, 187 (2003).

[4] H.D. Young e R.A. Freedman, Física II: Termodinâmica e Ondas (Addison Wesley, São Paulo, 2003).

[5] D. Halliday, R. Resnick e J. Walker, Fundamentos de Física (LTC, Rio de Janeiro, 2009), $8^{\text {a }}$ ed., v. 2.

[6] P.G. Hewitt, Física Conceitual (Bookman, São Paulo, 2015), $12^{\mathrm{a}}$ ed.

[7] V.L.B. de Jesus e D.A. Palma, Rev. Bras. Ens. Fís. 30, 3302 (2008).

[8] A. Garcia, D. Gonçalves, I. Perri, M.L. Campanari e M. Pegoraro, Estudo da densidade de fluidos incompressiveis através de um tubo em $U$, disponível em http://www.sorocaba.unesp.br/Home/Extensao/ Engenhocas/eurekarelatoriofinal.pdf acessado em $17 / 07 / 2018$

[9] M.A. Moreira, A teoria da aprendizagem significativa e sua implementação em sala de aula (UnB, Brasília, 2006).

[10] M.A. Moreira, O que é afinal aprendizagem significativa, disponível em http://moreira.if. ufrgs.br/oqueeafinal.pdf, acesso em 10/07/2018.

[11] BRASIL, Parâmetros Curriculares Nacionais: Ensino Médio (Ministério da Educação, Brasília, 2000). 